\title{
Ausblick
}

In dieser Untersuchung wurden Aporien polizeilicher Präventionsarbeit emergiert. Darauf aufbauend wurden mit einer theoretischen Kontextualisierung der empirischen Ergebnisse Konturen einer Professionalitätstheorie polizeilicher Präventionsarbeit beschrieben. Diese Umrisse bedürfen einer weiteren Ausdifferenzierung. Anregungen dazu wurden bereits in den Beiträgen zur Theoriebildung (vgl. Abschnitte 6.3.3 und 6.4) formuliert. Weitere Impulse werden in diesem Kapitel als Anregungen für die Forschung (Abschnitt 7.1) herausgestellt. Daran schließen sich Anregungen für die Praxis an (Abschnitt 7.2).

\subsection{Anregungen für die weitere Forschung}

Die durch diese Untersuchung zu beantwortenden Forschungsfragen sind erziehungswissenschaftlicher Natur. Das systemübergreifende Zusammenspiel von pädagogischem Handeln und polizeilicher Praxis führt natürlich zu Ergebnissen, die über erziehungswissenschaftliche Diskurse hinausragen. Das soll nicht unerwähnt bleiben und findet deshalb in diesem Ausblick Berücksichtigung.

Nach Impulsen für weitere erziehungswissenschaftliche Arbeiten werden daher Anregungen für die Polizeiforschung und die Präventionsforschung skizziert. Weil an diesen Forschungsstand anknüpfende empirische Untersuchungen weitere professionstheoretische Überlegungen sowie Kooperations- und Präventionsforschung nicht nur für eine Wissenschaftsdisziplin Erkenntnisse erwarten lassen, wird auf eine Zuordnungen der Anregungen zu einzelnen Wissenschaftsdisziplinen verzichtet. 
Empirische Anschlussuntersuchungen

In dieser Forschungsarbeit wurden Handlungsorientierungen von Polizeibeamtinnen und Polizeibeamten rekonstruiert und auf der Ebene einer Sinngenese maximal kontrastive Orientierungstypen gebildet (vgl. Abschnitt 5.4). Daran könnten sich Arbeiten anschließen, die sich mit den Entstehungsbedingungen dieser Orientierungen befassen (Soziogenese). Es deutet sich bereits in dieser Untersuchung die Relevanz normativer, berufsbiografischer, konzeptioneller und organisationskultureller Einflussfaktoren an (vgl. Abschnitt 5.5). ${ }^{1}$

Quantitative Untersuchungen könnten die Hypothesen dieser Untersuchung überprüfen und weitere empirische Erkenntnisse gewinnen. So dürften Häufigkeiten, regionale Verteilungen und institutionelle Zusammenhänge Aufschluss über die Relevanz der drei Orientierungstypen für die weitere Theoriebildung und für die Steuerung der Praxis geben.

Jenseits von Evaluationsforschung, die aus einer anderen Perspektive einen Blick auf die Präventionsarbeit wirft, existieren mit dieser Forschung Selbstbilder der polizeilichen Präventionsarbeit und deren Blick auf relevante Andere wie Kinder, Eltern, Lehrkräfte, Sozialarbeiterinnen und Sozialarbeiter. Für die weitere Theoriebildung könnte deren Perspektive auf die polizeiliche Präventionsarbeit von Bedeutung sein.

Erforscht wurde die polizeiliche Arbeit mit Kindern und Jugendlichen. Die polizeiliche Verkehrssicherheitsarbeit und Kriminalprävention hat aber weitere Zielgruppen, wie z. B. Senioren im Straßenverkehr oder Zuwanderinnen und Zuwanderer aus anderen Ländern. Diese Arbeitsfelder können empirisch mit erwachsenenpädagogischen Fragestellungen untersucht werden.

\section{Interdisziplinäre Professionsforschung}

Die polizeiliche Präventionsarbeit zeigt, dass in diesem Handlungsfeld pädagogisches Denken und Handeln nicht nur auf pädagogisch professionelle Akteurinnen und Akteure oder Bildungsinstitutionen begrenzt ist. Die Erziehungswissenschaft muss deutlicher als bisher zur Kenntnis nehmen, dass professionell gerahmtes pädagogisches Handeln auch jenseits genuin pädagogischer Professionalität stattfindet. Diese soziale Tatsache zu negieren, würde bedeuten non-formale Bildungssettings aus einer entsprechenden wissenschaftlichen Betrachtung zu exkludieren. Das gilt insbesondere für die Schulpädagogik. Es sind ja nicht nur Präventionsbeamtinnen und Präventionsbeamte der Polizei, die an Unterrichten

\footnotetext{
${ }^{1}$ Zur Relevanz von Berufsbiografien und organisationalen normativen Erwartungen in der praxeologischen Professionalisierungsforschung siehe Bohnsack (2020, S. 69 ff.).
} 
mitwirken, sondern Akteurinnen und Akteure ganz unterschiedlicher zivilgesellschaftlicher Institutionen, wie NRO-Mitarbeitende (vgl. Eich 2021) oder Religionspädagoginnen und Religionspädagogen im Kirchendienst (vgl. Endres 2019). Selbstständiges Handeln nicht hauptamtlicher Lehrpersonen im Schulunterricht wäre unvertretbar, wenn dieser Praxis nicht eine pädagogische Qualität beigemessen würde.

Die Institutionen der Polizei und die polizeibezogene Präventionsforschung kommen dagegen nicht umhin, sich mit pädagogischer Theorie und der Eigenlogik pädagogischer Systeme auseinanderzusetzen. Präventionsarbeit darf als pädagogisches Handeln verstanden werden, weil es regelmäßig die Merkmale eines solchen Handelns in sich trägt. Von zentraler Bedeutung sind daher vor dem Hintergrund der empirischen Ergebnisse dieser Untersuchung die Vermittlung des Normsinns, die Ergebnisoffenheit angeregter Lernprozesse und insgesamt die pädagogischen Aporien dieses Handelns. Die Professionsforschung steht vor der Herausforderung, systemübergreifendes Handeln als ein mögliches Merkmal von Professionalität zu definieren.

Da polizeiliche Präventionsarbeit nicht nur an Schulen stattfindet, sind die Ergebnisse dieser Untersuchung ein Beleg dafür, dass die Erziehungswissenschaft weiterhin vor der Herausforderung steht, sich nicht nur dem Lernen in Bildungseinrichtungen, sondern verstärkt gesellschaftlichen Lernprozessen zu widmen (vgl. Lüders et al. 2010, S. 231). In diesem Zusammenhang sei auch auf den Diskurs zu Bildungslandschaften, insbesondere zu pädagogischem Handeln außerschulischer Professioneller an Schulen (vgl. Eich und Kepura i.E.) hingewiesen.

Vor dem Hintergrund der skizzierten Weite pädagogischer Settings und pädagogisch handelnder Akteurinnen und Akteure bedarf es auch einer erziehungswissenschaftlichen Überprüfung und Justierung der Grenzen pädagogischen Handelns (vgl. Lüders et al. 2010, S. 230). Es darf zu keiner Entgrenzung und einer damit gleichzeitig verbundenen Konturenlosigkeit pädagogischen Handelns und pädagogischer Professionalität kommen.

\section{Kooperationsforschung}

In der vorangegangenen Anregung für die weitere Theorieentwicklung wurden Impulse für den Diskurs zur pädagogischen Professionalität gegeben. Mit dieser Arbeit liegt der empirische Befund vor, dass sich die Akteurinnen und Akteure polizeilicher Präventionsarbeit an einer eigenständigen, impliziten professionellen Identität orientieren (vgl. Abschnitt 5.2). Daher stellt sich auch die Frage nach Anregungen für die Kooperationsforschung. 
Sowohl die Ergebnisse von Eich (2021, S. 240) zur Zusammenarbeit entwicklungspolitischer Nichtregierungsorganisationen (NRO) mit Schulen als auch die Ergebnisse dieser Untersuchung ,,lassen erkennen, dass institutionelle Kontexte der Zusammenarbeit einen Rahmen für die handlungsleitenden Orientierungen der (außerschulischen) Professionellen bilden. Die Ziele und Handlungsaufträge der Herkunftsinstitution bleiben auch in dem Handlungssetting Schule wirksam“ (Eich und Kepura i.E.). Daher verspricht es erkenntnisreich zu sein, ,professionelles Handeln in interprofessionellen Kooperationskontexten nicht nur in Hinblick auf die kooperierenden Professionellen und deren Verhältnis zu betrachten, sondern den Fokus auf institutionelle Rahmungen zu erweitern. Diese Überlegungen könnten dann auch den Diskurs um die Verhältnisbestimmung von Organisation und Profession im erziehungswissenschaftlichen Bereich bereichern" (Eich und Kepura i.E.).

In der schulpädagogischen Forschung findet die Zusammenarbeit zwischen Polizei und Schulen - im Gegensatz zur praktischen Relevanz - kaum Beachtung. Dagegen scheinen bei dem durchaus existenten Diskurs zur Kooperation zwischen Akteurinnen und Akteuren der Polizei und der Sozialen Arbeit deutlich divergierende Grundpositionen, unterschiedliche Aufgabenzuweisungen, berufsständische Interessen und Abgrenzungsbemühungen durch (vgl. Abschnitt 6.3.2). Der normativ geprägte Professionalitätsdiskurs zwischen Sozialer Arbeit und polizeilicher Arbeit mit Kindern und Jugendlichen zeigt, wie bedeutsam eine empirische Fundierung des professionsbezogenen Handelns in der Präventionsarbeit ist, zu der diese Untersuchung einen Beitrag leistet. Eine entsprechende Kooperationsforschung könnte sich anschließen.

\section{Erforschung der polizeilichen Praxis}

Von Protagonisten der Polizeiforschung wird ein mangelndes Interesse innerhalb der Polizei, insbesondere bei Entscheidungsträgern, an wissenschaftlichen Erkenntnissen und der Erforschung der Polizei beklagt (siehe Abschnitt 3.1). Das Zentrum der Kritik rankt sich um die in der Polizei überaus wirkmächtige praxeologische Verwertungslogik von Wissen. Polizeiliches Handeln speist sich im Wesentlichen aus dem Erfahrungswissen der Organisation. Darüber hinausgehende Wissensbestände werden nach dem unmittelbaren Nutzwert und der Integrationsfähigkeit tradierter polizeilicher Wissensbestände beurteilt. Die Ergebnisse der vorliegenden Untersuchung zeigen, dass die Konturierung einer Theorie der Praxis sowohl für die Theorieanreicherung als auch für praxisbezogene Handlungsempfehlungen Erkenntnisse liefern kann. Daher ist diese Forschungsausrichtung im besonderen Maße geeignet, in einem ganzheitlichen Sinne den Ansprüchen und Interessen gerecht $\mathrm{zu}$ werden, die von einer Erforschung der Polizeiarbeit erwartet werden können. An die Wissenschaft darf 
dabei der Anspruch gestellt werden, ihre Erkenntnisse so zu vermitteln, dass sie Praktikerinnen und Praktikern zugänglich werden und einen Praxistransfer ermöglichen. Auf diese Weise könnte eine Brücke über die vermeintlich weit auseinanderliegenden Ufer namens Theorie und Praxis geschlagen werden.

Während alltagssprachlich von der Polizei gesprochen wird, verbergen sich hinter diesem Rahmen facettenreiche Aufgaben und heterogene Handlungsfelder (vgl. z. B. Mokros 2010, S. 205). Bisherige qualitative Forschungen zu Handlungsorientierungen von Polizeibeamtinnen und Polizeibeamten ${ }^{2}$ zeigen, dass in den Handlungsfeldern wiederum kontrastreiche Orientierungsmuster der Akteurinnen und Akteure wirkmächtig sind. Wenn Praxisorientierung dem polizeilichen Selbstverständnis entspricht, kann durch die Erforschung der Praxis die Grundlage für eine empirisch fundierte Polizeitheorie geschaffen werden. Impulse zur Verkleinerung der bestehenden Forschungsdesiderate können von weiteren hypothesengenerierenden Untersuchungen ausgehen.

\section{Systematisierung von Forschungsständen der Polizeiforschung}

Die begrenzte Anzahl von empirischen Studien zum Handeln in den unterschiedlichen polizeilichen Arbeitsfeldern in Deutschland lässt nicht nur große Forschungsdesiderate, sondern auch die begrenzte Präsenz von Akteurinnen und Akteuren der öffentlichen Sicherheit als Forschungsgegenstand nationaler Forschungseinrichtungen erkennen. Neben wenigen Einrichtungen, die sich intensiver der Polizeiforschung widmen, ist der Erkenntnisstand weit über unterschiedliche wissenschaftliche Disziplinen verstreut (vgl. Initiative Polizei in der Wissenschaft 2018, S. 259). Umso mehr gilt das für interdisziplinär nutzbare Erkenntnisse. So liefert z. B. diese primär erziehungswissenschaftlich ausgerichtete Arbeit auch Erkenntnisse für die Soziale Arbeit, Polizeiforschung, Präventionsforschung, Kriminalistik, Kriminologie und Verkehrsunfallforschung. ${ }^{3}$ Aus diesem Befund ergibt sich zwangsläufig die Empfehlung einer Systematisierung der vorliegenden Erkenntnisse. Durch entsprechende Arbeiten würde das Forschungsfeld nicht nur relevanter und interessanter erscheinen, die Ermittlung prioritärer Forschungsvorhaben würde so leichter fallen.

\footnotetext{
${ }^{2}$ Beispiele: Mensching (2008) zu Führung und Organisationskultur in der Polizei. Asmus und Enke (2016) zum Umgang der Polizei mit migrantischen Opfern. Weiterhin: Die Ergebnisse dieser Untersuchung.

${ }^{3}$ Es wird nicht der Versuch unternommen, Einzelerkenntnisse dem einen oder anderen Forschungsgebiet zuzuweisen. Die Grenzen dürften fließend sein. Die Relevanz für die genannten Wissenschaftsdisziplinen wird unterschiedlich stark ausgeprägt sein.
} 


\section{Präventionsforschung}

„Präventionsarbeit systemübergreifend zu erforschen (hier: pädagogisches Handeln und polizeiliche Praxis), eröffnet größere Erkenntnisperspektiven als der theoretische Rahmen nur einer Wissenschaftsdisziplin, weil das generierte Wissen dann nicht in der Eigenlogik des jeweiligen Systems verhaftet bleibt. Der Ruf nach einer interdisziplinären Präventionsforschung (vgl. Steffen 2014, S. 71) wird durch diese Forschungsergebnisse unterstützt" (Kepura 2019, S. 10).

Wenn es um die Erforschung der Präventionspraxis geht, werden aktuell Wirksamkeits- und Messbarkeitsfragen betont (vgl. Hölterhoff 2016; Armborst 2019). Im Gegensatz zu dieser Outputorientierung scheint die Inputseite in der polizeibezogenen Präventionsforschung weniger Beachtung zu finden. Wenn schon die Präventionsarbeit im Lichte einer Evidenzbasierung der Ergebnisse in ökonomischen Kategorien von Effizienz und Effektivität gedacht wird, erscheint auch die Erforschung des individuellen Beitrags der Ressource Mensch und deren Handlungsorientierungen als Wirksamkeitsfaktor sinnvoll und weist gleichzeitig über das ökonomische Paradigma hinaus. Die bisherigen Anregungen für die Erforschung von Präventionsarbeit beinhalten insgesamt auch Impulse für die Präventionsforschung.

\subsection{Anregungen für die Praxis}

Die Anregungen für die Praxis zeigen Entwicklungspotenziale für die polizeiliche Präventionsarbeit und mithin auch entsprechender Qualifikationserfordernisse auf. Gemeinsam mit der theoretischen Fundierung der polizeilichen Präventionsarbeit (vgl. Abschnitt 6.4 und 7.1) können die Rolle der Polizei und die Handlungsgrundlagen der polizeilichen Präventionsarbeit deutlich stärker konturiert, fundiert und damit gestärkt werden.

\section{Generierung, Systematisierung und Fundierung von Handlungswissen}

Die empirischen Ergebnisse dieser Forschung haben sehr deutlich gezeigt, dass Präventionsarbeit leistende Beamtinnen und Beamte ihr Handlungswissen für diese Aufgabe primär aus der Handlungspraxis generieren. Bedeutsam sind hierbei neben beruflichen Vorerfahrungen und den impliziten Bildern von Kindheit und Jugend insbesondere die Erfahrungen des eigenen Handelns (vgl. Abschnitt 5.4). Diese praxeologischen Wissensbestände bilden den selbstreferenziellen Reflexionsrahmen. Die Spezifik der polizeilichen Präventionsarbeit liegt in 
der Kombination personifizierter polizeilicher Expertise und pädagogischen Handelns. ${ }^{4}$ Während polizeiliche Wissensbestände explizierbar vorhanden sind, wird die Handlungspraxis häufig von einer impliziten Alltagspädagogik (vgl. Schäffter 1999) angeleitet. Es bedarf einer handlungspraktischen Umsetzung der empirischen Erkenntnis dieser Untersuchung, dass auch polizeiliche Präventionsarbeit von pädagogischem Handeln geprägt ist. Hierzu ist in einem viel stärkeren Maße als bisher die Erschließung pädagogischen Wissens ${ }^{5}$ als Handlungsgrundlage und Reflexionswissen ${ }^{6}$ sowie zur Zielbestimmung erforderlich. Letztlich soll dieses Wissen auch dazu führen, mit den Aporien polizeilicher Präventionsarbeit balancierend umzugehen. Im Mittelpunkt steht hierbei der gleichzeitige Umgang mit den unterschiedlichen systemischen Eigenlogiken von pädagogischem Handeln und polizeilicher Praxis. ${ }^{7}$

\section{Weiterentwicklung einer partizipativen Kooperation}

Aus polizeilicher Sicht ist die Prävention zur Förderung der öffentlichen Sicherheit eine gesamtgesellschaftliche Aufgabe (vgl. Kapitel 2). Neben einem schulpädagogischen Diskurs zur Kooperation mit der Polizei und einer empirischen Fundierung und Differenzierung entsprechender programmatischer Positionierungen in der Sozialen Arbeit (vgl. Abschnitt 6.3.2) bedarf es einer Förderung und weiteren konzeptionellen Ausgestaltung der handlungspraktischen Zusammenarbeit unterschiedlicher Akteurinnen und Akteure. Für die primäre Präventionsarbeit bestehen Konzepte (vgl. Abschnitt 2.3) und Materialien (z. B. Medien der „Polizeilichen Kriminalprävention der Länder und des Bundes“), die Orientierung und Hilfen geben. In der Typologie dieser Forschungsarbeit sind jedoch ganz

\footnotetext{
${ }^{4}$ Bei Jugendsachbearbeiterinnen und Jugendsachbearbeitern werden teilweise sozialpädagogische Handlungsorientierungen sichtbar.

${ }^{5}$ Dazu gehört z. B. pädagogisches Grundlagenwissen, wie die Unverfügbarkeit von Menschen in Erziehungssituationen und eine damit einhergehende „Ungewissheit“ bezogen auf intendierte Lernerfolge. Es ist ein Transfer pädagogischer Logik auf das Handlungsfeld polizeilicher Präventionsarbeit erforderlich. Dazu gehört dann auch der Umgang mit der Erkenntnis, dass mit pädagogischem Handeln nicht unmittelbar polizeiliche Zwecke, sondern nur pädagogische Ziele erreicht werden können. Dieser Zusammenhang schließt ein mitgängiges Erreichen polizeilicher Präventionsziele nicht aus.

${ }^{6}$ Diese Herausforderung besteht aber nicht nur für Polizistinnen und Polizisten. „Die Reflexionsfähigkeit und -praxis von Pädagogen erfolgt möglicherweise auf der Basis von sedimentiertem, nicht zu explizierendem Wissen. Wenn dieser noch unsichere Befund sich erhärten sollte, dann steht die erziehungswissenschaftliche Professionalisierungsforschung vor der Bewältigung neuer inhaltlicher und forschungsmethodischer Herausforderungen“, Thole (2016, S. 525).

${ }^{7}$ Eigenlogiken: Pädagogisches Handeln - Förderung der Entwicklung (junger) Menschen. Polizeiliche Praxis - Förderung der Sicherheit der Gesellschaft.
} 
unterschiedliche Rollenbilder der Beamtinnen und Beamten in der Präventionsarbeit sichtbar geworden. Mit diesen heterogenen Selbstbildern korrespondieren unterschiedliche Bilder von genuin pädagogischem Handeln in der Präventionsarbeit und pädagogische Alltagskonzepte der Beamtinnen und Beamten. Es zeigt sich ein Bedarf an der Klärung von Rollenerwartungen und didaktischer Fragen sowie der damit einhergehenden professionsübergreifenden Verantwortung für die Präventionsarbeit. Keine Akteurinnen und Akteure, weder die Polizistinnen und Polizisten, noch die Pädagoginnen und Pädagogen, dürfen ihre Verantwortung an die jeweils andere Seite delegieren. Die vorliegenden Erkenntnisse dieser Arbeit indizieren eine Intensivierung partizipativer Zusammenarbeit, insbesondere durch die gemeinsame Gestaltung von Lehr-Lern-Arrangements.

Weiterhin erscheint es bedeutsam, ,normative Orientierungen von schulischen und außerschulischen Akteurinnen und Akteuren in Beziehung zu setzen und in der Praxis zu reflektieren, wie vor dem Hintergrund dieser Einflussgröße eine Zusammenarbeit von schulischen und außerschulischen Partnerinnen und Partnern gestaltet werden kann. Äußerst erkenntnisreich dürfte dabei auch die Fokussierung der didaktischen Wirkungen von Kooperationsarrangements sein sowie deren kritische Reflexion“" (Eich und Kepura i.E.).

In der Zusammenarbeit zwischen Polizei und Akteurinnen und Akteuren der Jugendhilfe scheint es weiterhin vordinglich zu sein, bestehende normativ kolorierte Vorbehalte gegenüber der jeweils anderen Seite zu überwinden und so Kooperation zu fördern (vgl. Abschnitt 6.3.2).

Die Heterogenität der Handlungsorientierungen der Beamtinnen und Beamten weist darauf hin, dass es nicht eine zentrale Empfehlung für die Entwicklung einer Zusammenarbeit der Akteurinnen und Akteure gibt, sondern zunächst immer individuelle Praktiken und Kooperationsformen reflektiert werden müssen.

\section{Überwindung von Handlungsgrenzen durch systemisches Denken und Handeln}

Polizeiliche Präventionsarbeit findet dort ihre Grenzen, wo genuin polizeiliche Repressionspflichten greifen. ${ }^{8}$ Während sich die unterschiedlichen Wirkungsrichtungen von Gefahrenabwehr und Strafverfolgung noch über die Figur der doppelfunktionalen Maßnahmen verbinden lassen (vgl. Abschnitt 6.3.1) ist das für die zwangsfreie Normvermittlung nicht möglich. In dieser Arbeit wurde herausgearbeitet, dass genuin polizeiliche Befugnisse auf die Durchsetzung von Normen ausgerichtet sind und daher keinen Werkzeugkasten für die polizeiliche Präventionsarbeit darstellen. Die Beamtinnen und Beamten greifen deshalb mehr oder weniger implizit auf ein pädagogisches Handlungsrepertoire zu. Insbesondere anhand des Typus pädagogisierte Präventionsarbeit konnte gezeigt

\footnotetext{
${ }^{8}$ Insbesondere: Strafverfolgungspflicht gem. § 163 Abs. 1 StPO.
} 
werden, dass die Bewältigung dieser Grenzen zu Irritationen und Unsicherheiten im Handeln führen kann (vgl. Abschnitt 5.3.2). Besonders augenfällig wird diese antinomische Handlungsstruktur bei Jugendsachbearbeiterinnen und Jugendsachbearbeitern. In der primär kriminalistischen Ermittlungsarbeit fallen repressive und präventive Arbeitsaspekte regelmäßig zusammen (vgl. Abschnitt 6.1). Der Umgang mit diesen Grenzen darf nicht von Zuständigkeitsfragen determiniert sein, sondern bedarf im systemischen Sinne einer Vernetzung von Kooperationspartnern (vgl. die Orientierungsmuster des Typus subjektorientierte Präventionsarbeit, vgl. Abschnitt 5.3.3). Mögen die unmittelbaren präventiven Handlungsoptionen von Polizistinnen und Polizisten begrenzt sein, so muss das handlungsorientierte Denken, insbesondere das pädagogische Denken, deutlich über diese Systemgrenze hinausreichen, um die Maßnahmen anderer Akteurinnen und Akteure zu initiieren, zu fördern und zu begleiten.

\section{Reflexion des Umgangs mit Macht}

Von den Akteurinnen und Akteuren einer Institution, die dazu legitimiert ist, ein bestimmtes Verhalten von Menschen zu erzwingen, und deren Handeln eng mit Sanktionen in Verbindung steht, könnten leicht Wirksamkeitserwartungen bezogen auf dieses Instrumentarium unterstellt werden. Wie in den Ergebnissen dieser Untersuchung bereits dargestellt wurde (vgl. Abschnitt 5.2), konnte eine ganz andere Homologie rekonstruiert werden. Keiner der Orientierungstypen vertraut auf die präventive Wirkung von Strafen oder erzieherischer Maßnahmen im Kontext des Jugendstrafrechts. Wonach gesucht wird, sind Modi der Durchsetzung oder der Vermittlung. Prävention soll wirksam sein. Fehlverhalten und die Opferwahrscheinlichkeit sollen reduziert werden, eine gesellschaftsadäquate Sozialisation soll erreicht werden. Auf die Wirksamkeit von Normverdeutlichung oder Normvermittlung kann man vertrauen oder darauf hoffen. Normdurchsetzung ist so nicht möglich. In diesem Zusammenhang setzen die Beamtinnen und Beamten in unterschiedlichen Modi Macht und Expertise ein. ${ }^{9}$ Die Wirkungserwartung genuin polizeilicher Maßnahmen ist in ihrer Grundstruktur punktueller

\footnotetext{
${ }^{9}$ Expertise: Felderkenntnis und Felderfahrungen, juristisches Wissen, Glaubwürdigkeit (weil der Umgang mit abweichendem Verhalten das Geschäft der Polizei ist).

Macht: Die Polizei hat die Befugnis, ein Handeln zwangsweise durchzusetzen. Sie wird unspezifisch als sanktionierende Institution wahrgenommen (formal verhängen grundsätzlich die Justiz oder Ahndungsbehörden Sanktionen). Aber: Der Polizei kann man sich nicht entziehen, sie ist rund um die Uhr präsent. Ihr Handeln in pädagogischen Situationen ist die Ausnahme. Es besteht die unspezifische Erwartung, dass die Polizei akute Probleme der öffentlichen Sicherheit final löst.
} 
Natur. In einer konkreten Situation soll eine andere Person handeln, etwas erdulden oder eine Handlung unterlassen. Einstellung und Motivation sind zunächst von untergeordneter Bedeutung. Natürlich sind Verhaltensgründe von Bedeutung, zur Ergründung von Tatmotivation oder zur Erstellung von Gefährdungsbewertungen, aber die Grundform polizeilichen Handelns ist nicht die Vermittlung, sondern die Durchsetzung von Regeln. Pädagogisch ist die Zielstruktur genau entgegengesetzt. Hier geht es um Wissen, Lernanregungen usw. aber keine spezifischen Vorgaben und Verhaltenserwartungen. Die Ergebnisse sind offen und von den Lernenden abhängig. ${ }^{10}$ Die Melange aus tatsächlicher polizeilicher Macht und subjektiver Machterwartungen der Bevölkerung an die Polizei wird in dieser Untersuchung typspezifisch, also in unterschiedlichen Modi der Präventionsarbeit sichtbar (vgl. Abschnitt 6.1). In dieser spezifischen Expertise und den Zwangsbefugnissen drückt sich der Unterschied zu anderen Akteurinnen und Akteuren in der Präventionsarbeit aus, insbesondere wird so die Differenz zu Pädagoginnen und Pädagogen sichtbar, denen diese Attribute fehlen. Pädagogische und polizeiliche Macht sind unterschiedliche Formen von Macht. Sie können nicht wahlweise in die jeweils andere Funktionslogik übertragen werden. Für die polizeilichen Akteurinnen und Akteure in der Präventionsarbeit besteht daher regelmäßig die Herausforderung, ihr Handeln mit Blick auf die explizite und implizite Ausübung von Macht pädagogisch zu reflektieren, um der Logik pädagogischen Handeln gerecht zu werden und Irritationen zu vermeiden.

\section{Konzeptionelle Klärungen: Normverdeutlichung oder Normvermittlung}

Trotz aller konzeptionellen Grundlagen und Diskurse um die Rolle und Bedeutung von polizeilicher Präventionsarbeit (vgl. Kapitel 2 und 3) wird durch die in dieser Untersuchung emergierten kollektiven Handlungsorientierungen ein weiter Interpretationsspielraum der handelnden Akteurinnen und Akteure sichtbar. „In den empirischen Daten dieser Forschungsarbeit wird eine Ambivalenz zwischen Normverdeutlichung und Normvermittlung deutlich. Damit wird sichtbar, dass die Professionalität von Personen in der Präventionsarbeit sehr viel stärker auf diese Herausforderung ausgerichtet werden sollte und es gilt, Möglichkeiten zum konstruktiven Umgang mit widersprüchlichen Handlungsanforderungen zu finden" (Kepura 2019, S. 9). Es ist den Beamtinnen und Beamten offensichtlich selbst überlassen, ob sie ihre Aufgaben eher normenverdeutlichend oder normenvermittelnd interpretieren. Beide Ausrichtungen sind sicherlich möglich. Sie

\footnotetext{
${ }^{10}$ Aber auch hier gibt es Ergebniserwartungen, z. B. in Prüfungssituationen, aber eben wegen der Freiheitsgrade ist die Verbindlichkeit deutlich geringer als bei genuin polizeilichem Handeln.
} 
haben jedoch für Strategie, Konzeptionierung und Führung in der Polizei weitreichende Folgen. Eine Polizei, die sich in pädagogische Situationen und Räume begibt, muss pädagogisch handeln. Alles andere führt nicht nur zu Widersprüchen auf der Handlungsebene, sondern letztlich auch zu falschen Ergebniserwartungen. Repressionsfreie Präventionsarbeit kann mit pädagogischem Handeln ausschließlich pädagogische Ziele erreichen (Normvermittlung). Es reicht nicht aus, auf unmittelbare Zwangsbefugnisse zu verzichten und kommunikativ belehrend $\mathrm{zu}$ agieren. Ist Abschreckung durch normenverdeutlichende Gespräche die gewählte Methode, bleibt das Handeln auf polizeilicher Ebene (Normverdeutlichung). Konsequenterweise müssten dann auch pädagogische Räume für die Polizei verschlossen bleiben. Polizeiliche Präventionsarbeit dürfte sich dann wohl darauf konzentrieren, ihre Aktivitäten aus genuin polizeilichen Situationen zu generieren, bzw. sie damit zu verbinden. Unschärfen in der Konzeptionierung fördern die Gefahr von Irritationen, wie sie insbesondere beim Typus pädagogisierte Präventionsarbeit sichtbar wurden. Die Ergebnisse dieser Arbeit geben daher nicht nur Hinweise für die Weiterentwicklung der unmittelbaren Handlungspraxis, sondern regen auch zu grundsätzlichen Klärungen an. „Der Bildungsaspekt im Hinblick auf Verhaltensbeeinflussung durch Normverdeutlichung bzw. Normvermittlung wird unterschätzt und muss deutlich stärker im Selbstverständnis der Polizei verankert werden“ (Kepura 2019, S. 10).

\section{Stärkere Konturierung einer spezifischen Rolle und eines Qualifikationsprofils}

Die polizeinahe Literatur beschreibt die Wahrnehmung der polizeilichen Präventionsarbeit und ihrer Akteurinnen und Akteure als defizitär (vgl. Abschnitt 6.3.1). Einzelne Diskurse im empirischen Material dieser Untersuchung weisen in die gleiche Richtung. ${ }^{11}$ Soweit von den Beamtinnen und Beamten in den erhobenen Begleitinformationen (vgl. Abschnitt 4.2.2) polizeiinterne Fortbildungen als Qualifikation erwähnt wurden, scheinen sie für die Handlungspraxis von untergeordneter Bedeutung zu sein. Es bedarf daher einer stärkeren Konturierung einer spezifischen Rolle in der Polizei und eines Qualifikationsprofils.

\footnotetext{
${ }^{11}$ Eine differenzierte Auswertung dieser Fundstellen war zur Klärung der Forschungsfrage dieser Arbeit nicht zwingend erforderlich.
} 
Open Access Dieses Kapitel wird unter der Creative Commons Namensnennung 4.0 International Lizenz (http://creativecommons.org/licenses/by/4.0/deed.de) veröffentlicht, welche die Nutzung, Vervielfältigung, Bearbeitung, Verbreitung und Wiedergabe in jeglichem Medium und Format erlaubt, sofern Sie den/die ursprünglichen Autor(en) und die Quelle ordnungsgemäß nennen, einen Link zur Creative Commons Lizenz beifügen und angeben, ob Änderungen vorgenommen wurden.

Die in diesem Kapitel enthaltenen Bilder und sonstiges Drittmaterial unterliegen ebenfalls der genannten Creative Commons Lizenz, sofern sich aus der Abbildungslegende nichts anderes ergibt. Sofern das betreffende Material nicht unter der genannten Creative Commons Lizenz steht und die betreffende Handlung nicht nach gesetzlichen Vorschriften erlaubt ist, ist für die oben aufgeführten Weiterverwendungen des Materials die Einwilligung des jeweiligen Rechteinhabers einzuholen.

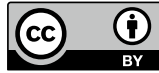

